\title{
Corrigendum to "A review of effect sizes and their confidence intervals, Part I: The Cohen's $d$ family”
}

\author{
Jean-Christophe Goulet-Pelletier ${ }^{\mathrm{a}, \bigotimes}$ and Denis Cousineau $^{\mathrm{a}}$ \\ ${ }^{\mathrm{a}}$ Université d’Ottawa
}

\begin{abstract}
Two errors have been found in the published article that are corrected here. All the simulations reported were error-free.

Keywords $\square$ Effect size, standard error, confidence intervals, Cohen’s $d$.

\section{jgoul014@uottawa.ca}

JCGP: 0000-0002-2016-549X; DC: 0000-0001-5908-0402

\subsection{2/tqmp.15.1.p054}

Two errors have been identified in the published article Goulet-Pelletier and Cousineau (2018). First, the $d_{D c}$ formula in Table 1, page 244, is erroneous. The correct formula is

$$
d_{D c}=d_{D} \times \sqrt{2(1-r)}
$$

(the division sign is replaced by a multiplication sign). This error is found within the text, on page 252, right column, first paragraph, with the incorrect sentence

$$
\begin{aligned}
& \text { the result of } d_{D} \text { is } \sqrt{2(1-r)} \text { times larger than } \\
& \text { Cohen's } d_{p}
\end{aligned}
$$

(the symbol $d_{D}$ and $d_{p}$ replaces $S_{D}$ and $S_{p}$ ). The mistake comes from the fact that the factor of conversion is incorrectly applied to the effect size estimate instead of its standard deviation. Therefore, when applied to the Cohen's $d$ effect size, the factor of conversion is $1 / \sqrt{2(1-r)}$. Hence, the equation (12a), at page 252 , should be

$$
d_{D}=d_{p} / \sqrt{2(1-r)}
$$

and equation (12b) on page 252 should be

$$
d_{p}=d_{D} \times \sqrt{2(1-r)}
$$

(the multiplication sign is replaced by a division sign and vice versa). Note that the simulations have been conducted using the correct formulas (12a) and (12b). Therefore, the results are not affected by this change. The mistake was introduced at the writing phase of the manuscript.

Second, regarding the standard error of Cohen's $d_{1}$ on page 256, left column, last paragraph, we omitted to mention that the standard error of $d_{1}$ is obtained by changing all " $2 / n$ " in Table 3 by " $1 / n$ ". Again, the simulations have been conducted using the correct standard error formula (using $1 / n$ ) for $d_{1}$. Therefore, the results are not affected by this change.

\section{Authors' note}

We are thankful to Louis Laurencelle and Bertram Walter who brought those mistakes to our attention.

\section{References}

Goulet-Pelletier, J.-C., \& Cousineau, D. (2018). A review of effect sizes and their confidence intervals, Part I: The Cohen's d family. The Quantitative Methods for Psychology, 14(4), 242-265. doi:10.20982/tqmp.14.4.p242

\section{Citation}

Goulet-Pelletier, J.-C., \& Cousineau, D. (2019). Corrigendum to “A review of effect sizes and their confidence intervals, part I: The Cohen's $d$ family”. The Quantitative Methods for Psychology, 15(1), 54-54. doi:10.20982/tqmp.15.1.p054 
publication in this journal is cited, in accordance with accepted academic practice. No use, distribution or reproduction is permitted which does not comply with these terms.

Received: 17/03/2019 Accepted: 17/03/2019 